\title{
Efficient implementation of complementary Golay sequences for PAR reduction and forward error correction in OFDM-based WLAN systems
}

\author{
Víctor P. Gil Jiménez*, M. Julia Fernández-Getino García, Matilde P. Sánchez-Fernández, \\ Ana García Armada \\ Department of Signal Theory and Communications, University Carlos III de Madrid, P.O. Box 28911 Leganés, Madrid, Spain
}

\begin{abstract}
In this paper the use of complementary Golay sequences (CGS) for peak-to-average power ratio (PAR) reduction and forward error correction (FEC) in an orthogonal frequency division multiplexing (OFDM)-based wireless local area network (WLAN) system is explored; performance is examined and complexity issues are analyzed. We study their PAR reduction performance depending on sequence lengths and we have found that, for the case that the number of sub-carriers differs from the sequence length, some interesting relationships can still be stated. Regarding their error correction capabilities, these sequences are investigated considering M-PSK constellations applied to the OFDM signal specified in IEEE 802.11a standard. Computational load for both Golay encoding and decoding processes is addressed and we provide an exhaustive analysis of their complexity. In order to overcome memory restrictions and speed up algorithmic operations, a novel algorithm for real-time generation of the Golay Base Sequences is proposed and evaluated giving as a conclusion that these sequences can be real-time generated with actual Digital Signal Processors (DSP). Our proposal lies on an efficient permutation algorithm that obtains the current permutation without the need for generating previous ones. Its complexity is calculated and turns out to be significantly low; the advantages are specially appreciated at the decoding stage. We also introduce a hybrid solution to get a trade-off between complexity and memory requirements. Moreover, the whole system is also implemented in a DSP to validate the proposal in a prototype, where its feasibility has been confirmed.
\end{abstract}

Keywords: OFDM; Complementary Golay sequences; PAR reduction; WLAN; FEC; Permutation generation

\section{Introduction}

Today's needs of bandwidth and flexibility are imposing the use of efficient modulations that are suitable for the characteristics of wireless channels. This is one of the reasons why multicarrier modulation techniques are increasingly used in wireless local area network (WLAN) environments. Standards, such as IEEE $802.11 \mathrm{a} / \mathrm{g}[1,2]$, have

\footnotetext{
* Corresponding author.

E-mail address: vgil@tsc.uc3m.es (V.P. Gil Jiménez).
}

chosen these technologies for high bit rate transmission and recent wider-range ones, such as IEEE 802.16a [3], are also adopting them in order to provide higher data rates. The choice of multicarrier technique orthogonal frequency division multiplexing (OFDM) is due to its good performance in multipath environments.

Basically, OFDM divides the available bandwidth into several orthogonal sub-carriers that can convey either data or control information. In order to maintain this orthogonality (which can be lost due to multipath effects) and eliminate the effects of inter-symbol interference (ISI), each OFDM 
symbol is cyclically extended at the beginning with the cyclic prefix. The design of an OFDM-system (symbol period, number of sub-carriers) should be carried out to guarantee the mitigation of the multipath channel effects. Indeed, this is achieved if the cyclic prefix is longer than the maximum delay spread of the channel. A careful choice of the number of sub-carriers and the cyclic prefix will ensure that the transfer function of each sub-channel is quasi-constant in time and frequency [4].

Keeping in mind that we would like the terminals to be low-cost, low-size and limited power consumption (mobile terminals need batteries' supply), it is desirable that physical layer requirements are not very demanding. The implementation of OFDM for WLAN systems offers great advantages due to the above-mentioned multipath performance, but it has also several critical aspects. Specifically, OFDM phase noise [5], time [6] and frequency synchronization [7] have been widely analyzed and new OFDM-based WLAN systems are being built, for instance, within European IST Program $[8,9]$.

However, one of the main disadvantages of OFDM is its high peak-to-average power ratio (PAR) which requires the use of linear amplifiers with a large dynamic range that are very power-inefficient and have an enormous impact on equipment's autonomy [10]. Thus, any research focused on reducing the PAR in OFDM signals is of high interest, specially that regarding WLAN scenarios for next generation networks [11].

For this reason, the literature is extensive and several methods have been already proposed, as for example, the use of complementary Golay sequences (CGS) [12], partial transmit sequences (PTS) [13,14], selective mapping (SLM) $[13,14]$, tone reservation [15], clustered transmission [16], orthogonal pilot sequences (OPS) [17] or pre-distortion techniques such as in [18].

Among all these possible solutions, CGS have been chosen in our study due to several reasons. First, using adequate parameters the signal PAR is bounded to $3 \mathrm{~dB}$ independently on the number of sub-carriers or characteristics of input data [12], unlike other techniques above. Second, the error correction capabilities of these codes would allow us to improve in some way the overall system performance through forward error correction (FEC). Third, the standard IEEE $802.11 \mathrm{~b} / \mathrm{g}$ $[2,19]$ employs complementary code keying (CCK) which are a sub-set of Golay sequences [20]. Finally, the number of sub-carriers that are required to efficiently combat multipath in WLAN environments is small (around 64 or 128 sub-carriers is enough), what makes the use of Golay sequences for PAR reduction an adequate choice due to the easiness and flexibility of their implementation in this case, as it will be shown later.

In this paper, the PAR reduction performance of CGS when the number of subcarriers either differs or not from the sequence length is analyzed, extending the analysis in [12], where only the equality case was examined. Besides, the error correction capabilities of these sequences consid- ering IEEE 802.11a parameters for the OFDM signal frame are studied, with a special emphasis on code rate effects. Additionally, complexity issues of Golay encoding/decoding operations are addressed to give an insight into their computational load.

To save memory requirements and avoid the use of lookup tables, a novel algorithm for real-time generation of the Golay base sequences is proposed, based on an efficient permutation algorithm. It shows low complexity and it is specially advantageous at decoding stages. It must be also pointed out that its loss in code rate efficiency is almost negligible. We also introduce a hybrid solution to get a trade-off between complexity and memory requirements for the cases when the number of sequences is very high and therefore complexity issues are not negligible anymore. Additionally, our proposed algorithm to generate Golay base sequences applies not only to OFDM but also to other systems using CGS such as CCK.

Finally, the whole system is also implemented in a digital signal processor (DSP) in order to validate the proposal in a prototype, where, as expected, physical real-valued signals exhibited a maximum PAR of $6 \mathrm{~dB}$ while simulated low-pass equivalent signals provided a maximum PAR of $3 \mathrm{~dB}$.

The remaining of this paper is organized as follows. In Section 2 a brief description of CGS is given and their characteristics and performance are analyzed; complexity issues are also examined. Then in Section 3 an algorithm for realtime generation of these Golay base sequences is proposed in order to avoid the use of a look-up table what leads to savings in memory. The physical implementation of the whole system and results are presented in Section 4 and, finally, in Section 5 some conclusions are drawn.

\section{Complementary Golay sequences for PAR reduction in OFDM}

In this section, the OFDM signal is presented and the discrete-time PAR is defined. Next, a brief description on CGS is shown in order to better understand why CGS are an alternative for PAR reduction in OFDM signals while they simultaneously provide forward error correction capabilities. Finally, the scope of our proposal is envisaged by addressing complexity issues of Golay encoding/decoding.

\subsection{OFDM and PAR}

OFDM divides the available bandwidth into $N$ orthogonal sub-carriers and each of them conveys a complex symbol. In a continuous transmission where several OFDM-symbols are transmitted, the time-domain sampled OFDM signal for the $\ell$ th OFDM symbol can be expressed as

$s^{\ell}[n]=\frac{1}{\sqrt{N}} \sum_{k=0}^{N-1} S^{\ell}(k) \mathrm{e}^{j \frac{2 \pi}{N} k n}, \quad 0 \leqslant n<N-1$, 
where $k$ and $n$ denote frequency and time indexes respectively, and $S^{\ell}(k)$ are complex data at $k$ th sub-carrier in frequency domain (e.g. M-PSK, M-QAM modulated). Assuming that complex data $S^{\ell}(k)$ are independent and identically distributed (i.i.d.), for a relatively large number of sub-carriers $(N \geqslant 32)$, the distribution of $s^{\ell}[n]$ $(0 \leqslant n<N-1)$ are near Gaussian random variables according to the central limit theorem. Thus, the discrete-time PAR for the $\ell$ th OFDM symbol is a random variable defined as

$\chi^{\ell}=\operatorname{PAR}\left\{s^{\ell}[n]\right\}=\frac{\max \left(\left|s^{\ell}[n]\right|^{2}\right)}{E\left\{\left|s^{\ell}[n]\right|^{2}\right\}}, \quad 0 \leqslant n<N-1$,

where $\max \left(\left|s^{\ell}[n]\right|^{2}\right)$ denotes the maximum instantaneous power of the signal, $E\left\{\left|s^{\ell}[n]\right|^{2}\right\}$ represents its average power in $N$ samples period and $E\{\cdot\}$ denotes the expected value. For certain OFDM symbols, input data may produce a highPAR signal. For this reason, it is common in the literature to denote the PAR of the signal as the maximum PAR over the whole transmitted signal, and thus, this is the definition used through the paper. In uncoded OFDM systems, when the number of sub-carriers $N$ is small, PAR depends on $N$ as $\operatorname{PAR}(\mathrm{dB}) \approx 10 \cdot \log N$. In [21] a further detailed analysis on PAR distribution is shown. However, if input data are adequately encoded with Golay sequences, PAR of the OFDM modulated signal is limited to $3 \mathrm{~dB}$ [12].

\subsection{Complementary Golay sequences}

Here, a brief description on CGS is provided and the relationships between PAR and CGS are hinted at. Davis and Jedwab introduced in [12] the use of CGS for PAR reduction, providing a complete theoretical study on them. They showed that it is possible to encode the original input data by using CGS in such a way that the obtained OFDM signal exhibits a PAR limited to $3 \mathrm{~dB}$. More exhaustive performance results on CGS are given in [22].

Two sequences $a$ and $b$ are CGS with respect to each other if the sum of their autocorrelations is null except in zero.

Let us define the following set of binary sequences $x_{i}$ parameterized by $m$, where $2^{m}$ is the sequence length:

$$
\begin{aligned}
& x_{1}=\{\overbrace{0000 \cdots 00}^{2^{m-1}} \overbrace{1111 \cdots 11}^{2^{m-1}}\} \\
& x_{2}=\{\overbrace{0 \cdots 0}^{2^{m-2}} \overbrace{1 \cdots 1}^{2^{m-2}} \overbrace{0 \cdots 0}^{2^{m-2}} \overbrace{1 \cdots 1}^{2^{m-2}}\} \\
& x_{m}=\{\overbrace{0101010101 \cdots 0101}^{2^{m}}\} \\
& 2^{m} \\
& x_{m+1}=\{\overbrace{111111111 \cdots 1111}\} .
\end{aligned}
$$

We also define a permutation $\pi$ on the set $\{1 \ldots m\}$, where $\pi_{k}$ represents the $k$ th element of the permuted set, i.e. there are $m$ ! possible permutations, where $(\cdot)$ ! denotes factorial of $(\cdot)$. If we fix the constellation size by the parameter $h$
( $2^{h} \mathrm{PSK}$ ) with $h>1$, there exist $m ! / 2$ complementary Golay sequences of the form [12] ${ }^{1}$ :

$2^{h-1} \sum_{k=1}^{m-1} x_{\pi_{k}} \odot x_{\pi_{k+1}}$,

that comprise one of $2^{h(m+1)}$ possible Golay sequences over $\mathbb{Z}_{2^{h}}$, the $\odot$ operation should be understood as the binary AND function.

Given a sequence of information bits, let us state a sequence in the form:

$C=\sum_{q=1}^{m+1} c_{q} x_{q}$,

where $c_{q} \in \mathbb{Z}_{2^{h}}$, are the values of a group of $h$ information bits expressed in decimal. Given a CGS in the form of Eq. (4) that we will denote as Golay base sequences (GBS) in the encoding process, if we combine it with another one constructed as described above in Eq. (5), it yields another CGS

$2^{h-1} \sum_{k=1}^{m-1} x_{\pi_{k}} \odot x_{\pi_{k+1}} \oplus \sum_{q=1}^{m+1} c_{q} \odot x_{q}$,

where the operator $\oplus$ denotes the addition operation in modulo $2^{h}$. The fact that the sequence in Eq. (6) is a CGS still holds for any permutation $\pi$ and any set of information bits defining sequence $C$. Eq. (6) defines the encoding process for a sequence of information bits into a CGS [12]: the bits to be encoded are divided into groups of $w+h(m+1)$ bits, of which $w$ parameter, known as base size, will select the GBS to be used and the others are grouped into $m+1$ sets of $h$ bits each one, and they will form $c_{q}$. Because there are only $m ! / 2$ GBS in the form of Eq. (4), there is a constraint in the values of $w$, i.e. $2^{w} \leqslant m ! / 2$. As an example, the GBS for $m=3$ and $h=4$ are [00080080, 00080800, 00000880], since in this case the number of GBS is $m ! / 2=3$.

Once the whole sequence is obtained, it is mapped into the proper $2^{h}$ PSK constellation in order to obtain $S^{\ell}(k)$. At the receiver side, the fast Hadamard transform (FHT) [23] is used for the decoding process [24,25].

Hence, there are $w+h(m+1)$ and $2^{m} h$ input and output bits respectively, and thus, the FEC code rate (defined as the ratio of input to output bits) is:

$R=\frac{w+h(m+1)}{2^{m} h}$.

As the efficiency of the code increases as $w$ does, it is usually fixed to $w=\left\lfloor\log _{2} m ! / 2\right\rfloor$, where $\lfloor x\rfloor$ operator denotes the integer smaller than $x$. For illustration, the values of $R$ depending on $m$ and $h$ are plotted in Fig. 1, where the parameter $w$ has been always fixed to its maximum value, i.e. $w=\left\lfloor\log _{2} m ! / 2\right\rfloor$. It can be seen that the main parameter from

\footnotetext{
${ }^{1}$ There only exist $m ! / 2$ sequences because symmetrical permutations generate the same sequence.
} 


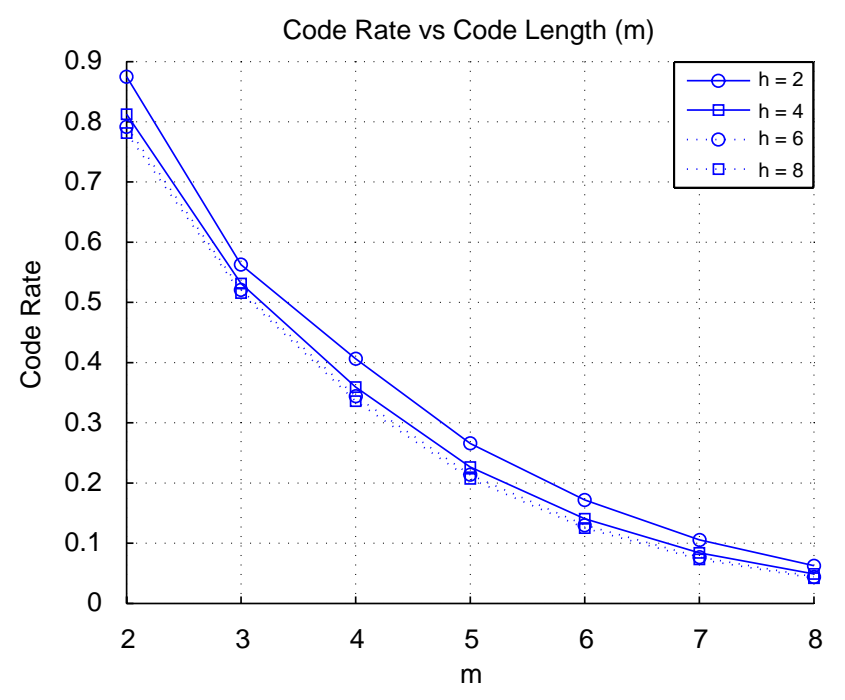

Fig. 1. Evolution of code rate with $\mathbf{m}$ and $\mathbf{h}$.

the code rate point of view is $m$ in an inverse relationship, i.e. the larger $m$ the lower the code rate is.

These CGS were originally proposed for phase modulation, this is, M-PSK constellations, but recently in [26] or [27] their use has been extended to 8-QAM and 16-QAM modulations. However, in this case the way of encoding is not yet defined and PAR depends on the chosen sequences.

\subsection{Performance of an OFDM-based WLAN system encoded with Golay sequences}

In the following, the performance of an encoded OFDM system by using CGS in terms of PAR reduction and Bit Error Rate (BER) will be studied for a WLAN environment, i.e. the OFDM signal frame and cyclic prefix will be set accordingly to match the IEEE 802.11a standard [1]. The number of sub-carriers will be varied around typical values for WLAN in order to understand the influence of this parameter.

\subsubsection{PAR and number of sub-carriers}

If the number of sub-carriers is equal to the sequence length, i.e. $N=2^{m}$, the PAR of the encoded OFDM system is limited to $3 \mathrm{~dB}$ [12]. In order to evaluate the behavior if the sequence length is different from $N$, several scenarios have been simulated with $N=2^{m \pm 1}$ and $N=2^{m \pm 2}$. The code length $(m)$ was fixed to 6 and 7, that for the case $N=2^{m}$ yields $N=64,128$ which are typical values for the number of sub-carriers in WLAN environments. Then, maintaining these values for $m$, we evaluated different number of subcarriers. In all these scenarios $w$ parameter has been fixed to its maximum value, i.e. $w=8$ and $w=11$ for $m=6$ and 7 , respectively, in order to maximize the code rate (maximizing code rate means the less throughput loss but the same error correction capabilities).
Table 1. PAR values depending on the number of sub-carriers $\mathbf{N}$

\begin{tabular}{|c|c|c|c|c|c|}
\hline$N$ & $m$ & $h$ & Relationship & Golay PAR (dB) & PAR $(\mathrm{dB})$ \\
\hline 16 & 6 & 2 & $2^{m-2}$ & 3.01 & 9.9 \\
\hline 16 & 6 & 3 & $2^{m-2}$ & 3.01 & 9.9 \\
\hline 16 & 6 & 4 & $2^{m-2}$ & 3.01 & 9.9 \\
\hline 32 & 6 & 2 & $2^{m-1}$ & 3.05 & 10.2 \\
\hline 32 & 6 & 3 & $2^{m-1}$ & 3.01 & 10.2 \\
\hline 32 & 6 & 4 & $2^{m-1}$ & 3.01 & 10.2 \\
\hline 64 & 6 & 2 & $2^{m}$ & 3.04 & 10.5 \\
\hline 64 & 6 & 3 & $2^{m}$ & 3.03 & 10.5 \\
\hline 64 & 6 & 4 & $2^{m}$ & 3.01 & 10.5 \\
\hline 128 & 6 & 2 & $2^{m+1}$ & 6.02 & 10.8 \\
\hline 128 & 6 & 3 & $2^{m+1}$ & 6.02 & 10.8 \\
\hline 128 & 6 & 4 & $2^{m+1}$ & 5.98 & 10.8 \\
\hline 256 & 6 & 2 & $2^{m+2}$ & 8.37 & 11 \\
\hline 256 & 6 & 3 & $2^{m+2}$ & 8.53 & 11 \\
\hline 256 & 6 & 4 & $2^{m+2}$ & 8.51 & 11 \\
\hline 32 & 7 & 2 & $2^{m-2}$ & 3.02 & 10.2 \\
\hline 32 & 7 & 3 & $2^{m-2}$ & 3.51 & 10.2 \\
\hline 32 & 7 & 4 & $2^{m-2}$ & 3.01 & 10.2 \\
\hline 64 & 7 & 2 & $2^{m-1}$ & 3.05 & 10.5 \\
\hline 64 & 7 & 3 & $2^{m-1}$ & 3.01 & 10.5 \\
\hline 64 & 7 & 4 & $2^{m-1}$ & 3.01 & 10.5 \\
\hline 128 & 7 & 2 & $2^{m}$ & 3.01 & 10.8 \\
\hline 128 & 7 & 3 & $2^{m}$ & 3.01 & 10.8 \\
\hline 128 & 7 & 4 & $2^{m}$ & 3.01 & 10.8 \\
\hline 256 & 7 & 2 & $2^{m+1}$ & 6.02 & 11 \\
\hline 256 & 7 & 3 & $2^{m+1}$ & 5.98 & 11 \\
\hline 256 & 7 & 4 & $2^{m+1}$ & 5.98 & 11 \\
\hline 512 & 7 & 2 & $2^{m+2}$ & 8.64 & 11.2 \\
\hline 512 & 7 & 3 & $2^{m+2}$ & 8.62 & 11.2 \\
\hline 512 & 7 & 4 & $2^{m+2}$ & 8.65 & 11.2 \\
\hline
\end{tabular}

In Table 1, a comparison for the PAR reduction using Golay codes is shown; PAR values provided in this table refer to maximum values over a continuous transmission of 10,000 OFDM symbols. It can be seen that when $N=2^{m}$ the PAR is $3 \mathrm{~dB}$, as proved in [12]. To extend this previous work, we have analyzed the case $N \neq 2^{m}$ and we found that if the sequence length is a multiple of the number of subcarriers (i.e. $N=2^{m-2}, 2^{m-1}$ ) the PAR is still $3 \mathrm{~dB}$. On the other hand, if the sequence length is half or a quarter of the number of sub-carriers (i.e. $N=2^{m+1}, 2^{m+2}$ ), the PAR is 6 and $8.5 \mathrm{~dB}$ respectively. In all cases the encoded OFDMsystem obtains lower PAR in several $\mathrm{dB}$ than the uncoded system (and PAR reductions are still similar to other schemes in the literature [13,15-17]).

Results in Table 1 point out that there is a certain flexibility in the design, because it is possible to select different coding schemes according to our needs keeping the signal PAR 


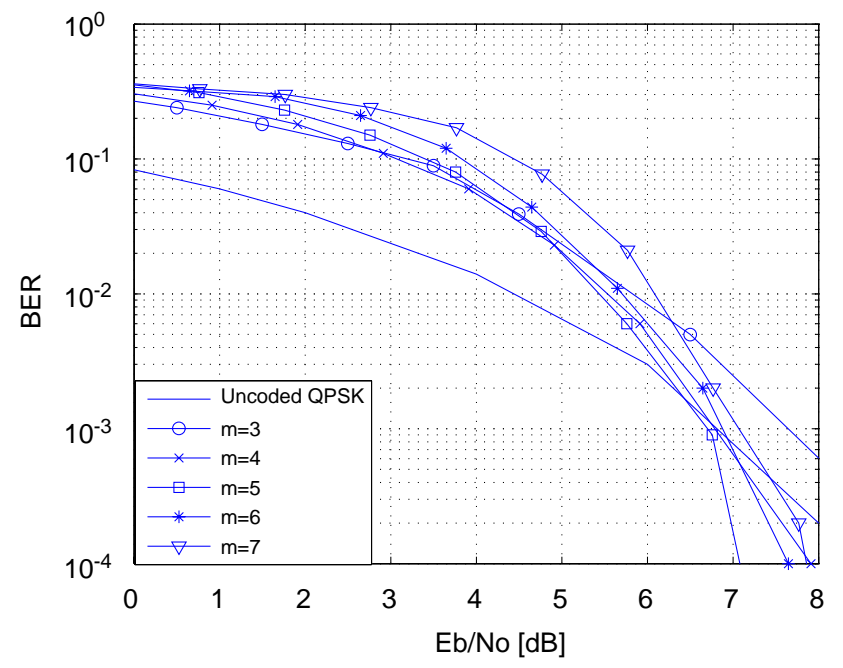

Fig. 2. BER vs sequence length parameter $\mathbf{m}$ for a QPSK constellation.

bounded to 3, 6 or $8.5 \mathrm{~dB}$ (different coding schemes provide different FEC capabilities as it will be shown).

\subsubsection{FEC performance}

Besides the PAR bounding characteristics of the CGS, they also provide FEC due to the relationship of these sequences with Reed Muller codes [12]. Therefore, combining the PAR reduction and FEC capabilities the overall system performance can be increased. The PAR reduction sets the system in a signal-to-noise ratio where the sequences provide FEC. In order to evaluate this kind of error correction, different scenarios have been simulated. We fixed $N=2^{m}$ and 10, 000 OFDM-symbols over an additive white Gaussian noise (AWGN) channel were simulated to obtain accurate results.

As it was shown in Section 2.2 the main parameter from the code rate point of view is $m$. In order to see how this parameter influences the performance, different simulations have been carried out keeping $h=2$, i.e. QPSK modulation, and $w$ at its maximum. In Fig. 2 it can be seen how these Golay sequences outperform the uncoded system over an $E_{b} / N_{0}=7 \mathrm{~dB}$. Another aspect that can be observed is that all the curves perform similar even though those with larger sequence length $(m)$ perform slightly better. It should be noted that $E_{b} / N_{0}$ in curves of Figs. $2-4$ is the coded $E_{b} / N_{0}$, i.e.

$\left(\frac{E_{b}}{N_{0}}\right)_{\text {coded }}[\mathrm{dB}]=\left(\frac{E_{b}}{N_{0}}\right)_{\text {uncoded }}[\mathrm{dB}]-R[\mathrm{~dB}]$.

On the other hand, in order to check the effect of the constellation size $(h)$, in Figs. 3 and 4 the performance for different values of $h$ is examined. In Fig. 3 a comparison between QPSK and 8-PSK is shown for different sequence's

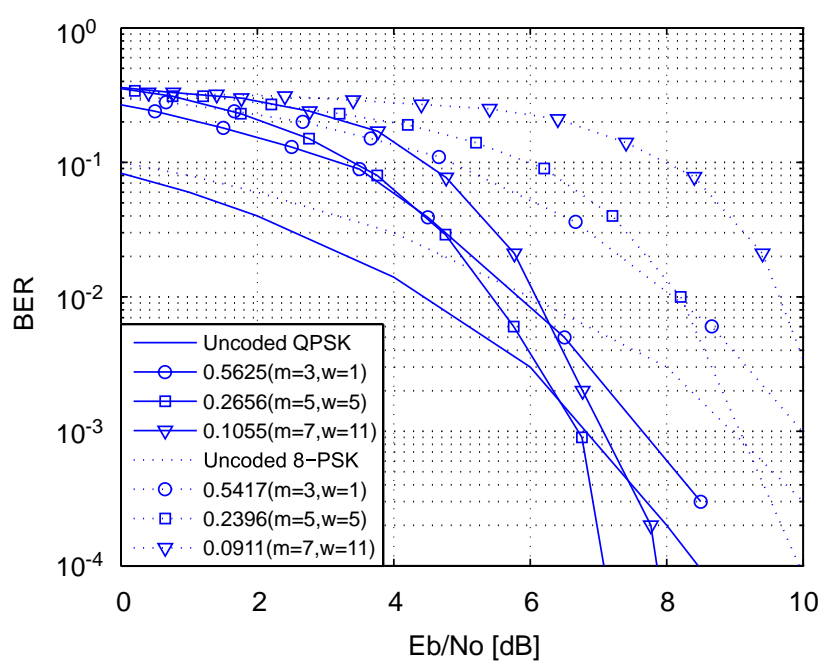

Fig. 3. Performance comparison between QPSK (solid line) and 8-PSK (dotted line).

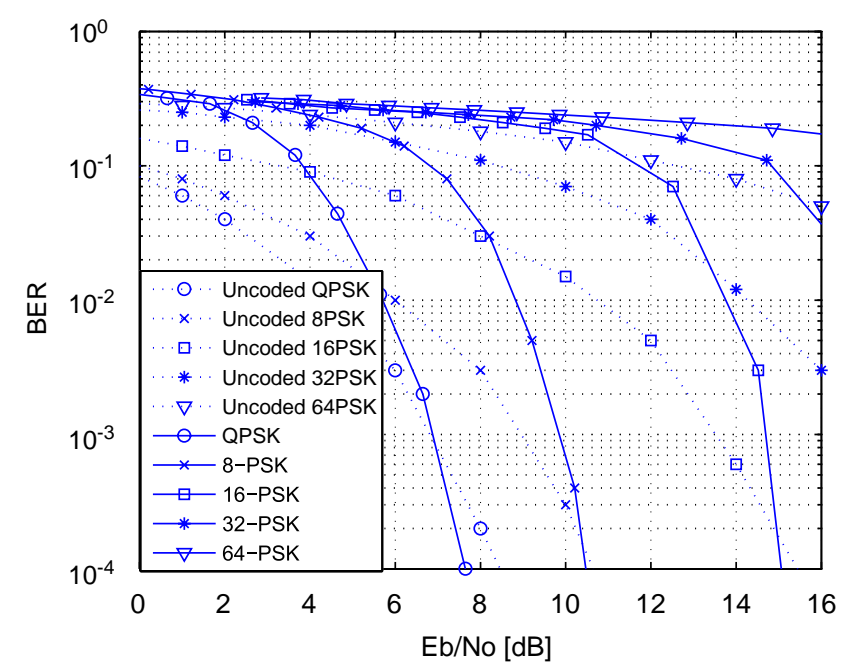

Fig. 4. Performance comparison for M-PSK constellations $(\mathbf{m}=\mathbf{6})$ for an uncoded (dotted line) and Golay encoded (solid line) system.

lengths. It can be seen that the effect of $m$ is more severe in the 8-PSK scheme than in QPSK because a larger value of $h$ decreases the code rate and increases constellation density.

In Fig. 4 the sequence's length parameter $(m)$ has been fixed to 6, i.e. $N=64$ (as in the IEEE 802.11a standard) and different values for $h$ have been explored. It can be seen that eventually the encoded system outperforms the uncoded one, and moreover, with the added value that obtained signal exhibits a PAR limited to $3 \mathrm{~dB}$. From both figures, it can be concluded that increasing the constellation size $(h)$ degrades code performance, even though the code rate is decreasing. The reason is that parameter $h$ only slightly affects the code rate, while $m$ is the dominant parameter (see Fig. 1). 


\subsection{Golay encoder/decoder complexity}

Once we have seen that CGS are useful for OFDM systems and in particular for WLAN environments, the complexity for encoding/decoding is now analyzed. Since physical devices are usually implemented on DSPs, the number of multiply add carry (MAC) operations is employed as a measurement. This operation takes two data and multiplies them, after that this result is added to another data and the final result is stored. Commonly, actual DSP are able to execute several MAC operations in one clock cycle and millions of them per second. The computational load will depend on the parameters: $m, h$ and $w$ but mainly on $m$ as we will show.

\subsubsection{Encoder complexity}

Assuming that all the GBS have been previously generated and stored into a look-up table, by examining the way of encoding them, there will be required:

- MAC operations: $2^{m}(m+2)$

- Divisions: $2^{m}$.

Thus, the encoder complexity only depends exponentially on $m$. It should be noted that, for the encoding process, only one GBS is used each time (that will be selected by the first $w$ bits).

\subsubsection{Decoder complexity}

On the other hand, the decoder does not always complete the same number of operations when decoding is FHTbased. The reason is that first, the GBS is estimated and then, it will depend on how many iterations are needed for a specific GBS. It will exist therefore a lower and an upper bound. Examining the algorithm, the upper-bound has been calculated, yielding:

- MAC operations: $\left(7 m \cdot 2^{m}+m^{2} \cdot 2^{m}+2^{m}+m^{4}\right) \cdot h+(h+1)$.

- Divisions: $2^{m} \cdot(1+m \cdot h+m ! / 2)$.

- Test operations: $\left(2^{m}+2\right) \cdot m+2^{w}$.

The test operations are comparisons between two numbers. It can be observed that the number of operations is much larger than in the encoder case and it depends exponentially on $m$ and $w$, and linearly on $h$. In this case $m$ and $w$ would affect the complexity much more than $h$. Nevertheless, this is the upper-bound which is rarely reached. In general, only a few sequences will be needed to be generated.

It would be interesting to compare the encoder and decoder complexity with respect to the complexity of the fast Fourier transform (FFT) implementation, since, on one hand, Inverse-FFT (IFFT) and encoder and, on the other hand, FFT and decoder, would be possibly implemented in the same DSP. For instance, the complexity of the complex radix 2 algorithm implemented in the TMS320C6201 DSP from Texas Instruments [28] is

$$
\begin{aligned}
\mathrm{NC}_{\text {radix } 2}= & \log _{2} N \cdot(7+2 N) \\
& +\frac{N}{4}+\left(\frac{N}{4}+2\right) 14+70 \text { cycles }
\end{aligned}
$$

where $N$ is the number of FFT points. It should be noted that Eq. (9) accounts for the three consecutive operations that are required to perform the FFT by using the radix 2 algorithm. ${ }^{2}$

In Fig. 5 the dependency on the number of cycles with respect to $m$ for the encoder and decoder is plotted (assuming one MAC per cycle ${ }^{3}$ ). The complexity for the radix-2 algorithm is also shown for comparison purposes. It can be observed that, in both cases, the Golay encoder/decoder has a reasonable complexity since it is in the range of the FFT. Actually, DSPs can execute several millions of instructions per second and therefore the Golay encoder/decoder seems to be feasible.

\section{Generation of base sequences}

One of the drawbacks of Golay sequences is their storage. If the sequence length, given by $m$, is high, the number of possible GBS may be too large for some applications and, moreover, this number increases exponentially with $m$ since $2^{w} \leqslant m ! / 2$.

\section{Permutation generator Algorithm (PA)}

1. Initialize array list with all elements in lexicographic order.

2. Initialize array permutation as empty, aux $=n, a c t=m$ and $i=1$, where $n$ is the number of the specific permutation, $m$ is the total number of elements to permute and $i$ will be the index for permutation array.

3. If $(n \bmod$ act! $)=0$, jump to 7 else jump to 4 .

4. $\quad$ res $=\frac{a u x}{(\text { act }-1) !}$. If res $>1$ res $=$ res -1 .

5. Set permutation $[i]=\operatorname{list}\left[(\text { res })_{2}\right]$. Delete $\operatorname{list}\left[(\text { res })_{2}\right]$ element from array list.

6. Update:

$$
\begin{aligned}
& \text { - } a c t=a c t-1 \\
& \text { - } a u x=a c t ! \cdot(\text { res }-(\text { res }) z) \\
& \text { - } i=i+1 \\
& \text { - If } i \leqslant m \text { go back to } 3 \text { else jump to } 7
\end{aligned}
$$

7. Complete array permutation with elements in array list in inverse order. The desired permutation is in the array permutation. Stop.

An approach to reduce the problem is limiting the number of valid GBS. The main advantage of this method is that

\footnotetext{
${ }^{2}$ In order to perform an FFT by using the radix 2 algorithm in the DSP library, a previous re-arrange of data is needed, and then, after radix 2 algorithm, data must be re-ordered again.

${ }^{3}$ Usually DSP are prepared to execute several MAC operations per cycle (depending on the pipeline architecture), and so, the actual number of cycles will be smaller.
} 

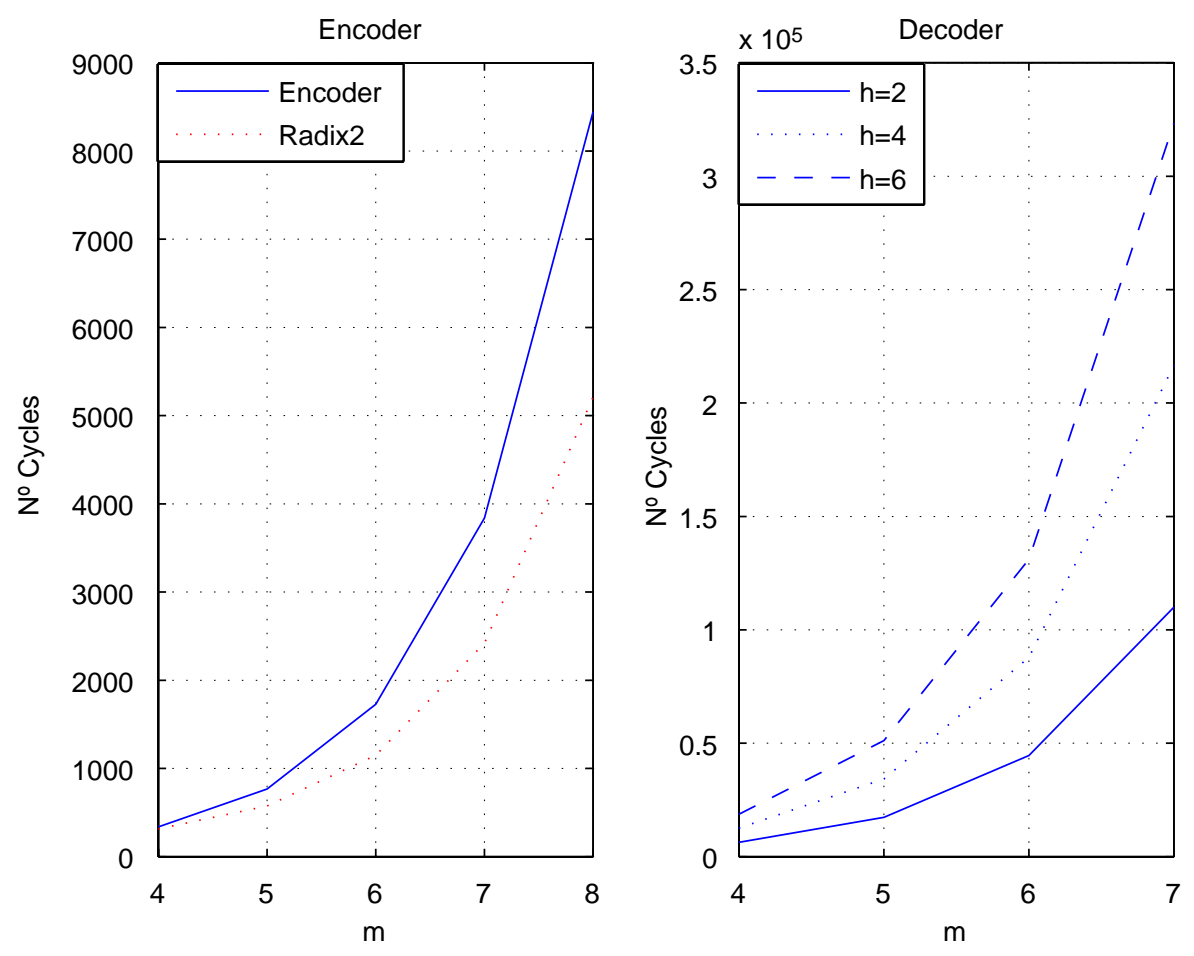

Fig. 5. Number of cycles for Golay encoder/decoder assuming one MAC operation per cycle.

all GBS can be analyzed and only the ones with best performance in terms of BER can be selected (this option has been chosen in the IEEE 802.11b standard [19]). However, this way we are reducing the code rate and therefore the performance, since it depends on $w$ as it was shown before. Next, a novel algorithm for real-time generation of GBS is proposed.

Examining Eq. (4), it can be observed that, if we are able to generate a specific permutation $\pi$, then the adequate GBS can be easily generated. The idea is based on sorting permutations in a known order, and then, being able to generate a specific one. Basically, there are two methods to generate all the permutations [29]: iterative and lexicographic algorithms. Both methods generate all the permutations for a given set. The iterative algorithm would generate the permutations pseudo-randomly, and the lexicographic would generate them in alphabetical order. However, if we want the $i$ th permutation both algorithms need to generate the previous $(i-1)$ permutations. This is, evidently, not useful for our purposes.

The lexicographic algorithm sorts permutations in a way that can be used for real-time generation. It should be noted that not all the $m$ ! permutations are valid but only half of them, because symmetrical permutations will generate the same GBS. Also, it would be interesting to know which permutations generate valid sequences. Looking at the structure of the lexicographic order it can be observed a factorial relationship in the form that first $\sum_{i=1}^{m-1}(m-i)$ ! permutations generate the first $\sum_{i=1}^{m-1}(m-i)$ ! valid GBS; the symmetri-
Table 2. Bit loss comparison table

\begin{tabular}{rrrcrl}
\hline$m$ & $m ! / 2$ & $w_{\max }$ & $\sum_{i=1}^{m-1}(m-i) !$ & $w_{\max }$ & Bit Loss \\
\hline 5 & 60 & 5 & 33 & 5 & 0 \\
6 & 360 & 8 & 153 & 7 & 1 \\
7 & 2520 & 11 & 873 & 9 & 2 \\
8 & 20160 & 14 & 5913 & 12 & 2 \\
9 & 181440 & 17 & 46233 & 15 & 2 \\
10 & 1814400 & 20 & 409113 & 18 & 2 \\
\hline
\end{tabular}

cal ones are above this range. By using this characteristic a Golay Base Sequences Generation Algorithm (GBSGA) has been designed. This algorithm makes use of the specific Permutation generator Algorithm (PA) whose pseudo-code is described above. In the pseudo-code mod denotes the modulo operation, $[j]$ represents the $j$ th element in an array and $(\cdot) 2$ takes the integer part of the number.

It can be seen that in this way it is not possible to obtain all the valid GBS but a set of them. However, Table 2 and Fig. 6 show that this loss is negligible. As it was pointed out in Section 2 the first $w$ bits select the GBS subject to $2^{w} \leqslant m ! / 2$, i.e. not all the $m ! / 2$ valid codes will be used. In Table 2 it can be seen that the number of available permutations with proposed algorithm, i.e. $\sum_{i=1}^{m-1}(m-i)$ !, are close enough to $2^{w}$ and therefore the loss due to the use of the algorithm is only 1 or 2 bits. For example, for $m=4$, the maximum value for $w$ is 3 . By using our algorithm it is possible to generate up to $\sum_{i=1}^{3}(4-i) !=9$ permutations, so it is still valid to 


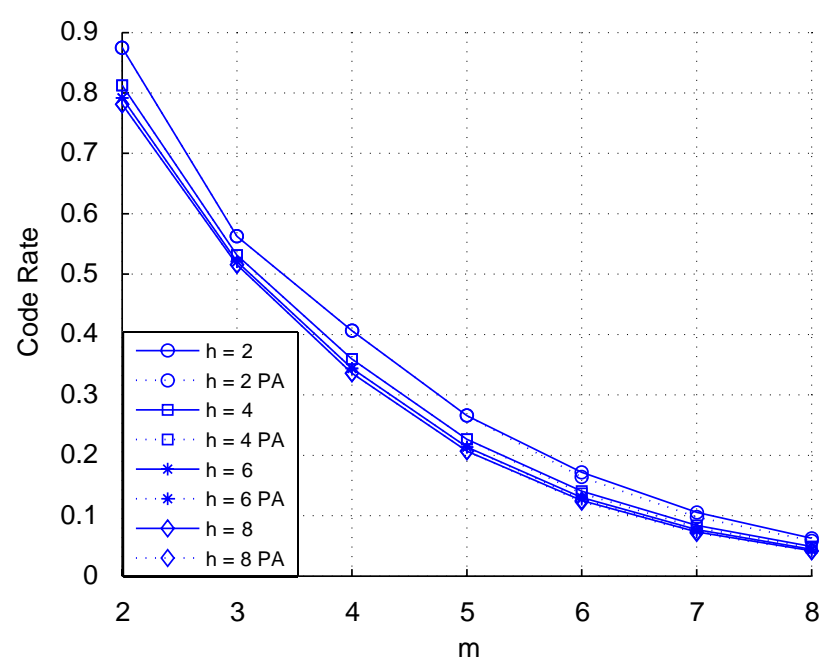

Fig. 6. Code-rate efficiency for different values of $\mathbf{h}$ without and with the Permutation Algorithm (PA).

Table 3. Memory needed to store GBS

\begin{tabular}{rr}
\hline$m$ & Memory (kBits) \\
\hline 5 & 1 \\
6 & 16 \\
7 & 256 \\
8 & 4096 \\
9 & 65535 \\
10 & 1048576 \\
\hline
\end{tabular}

set $w=3$. In Fig. 6 a comparison between the original code rate and once applied the algorithm is shown; it confirms that the loss in code rate after applying the PA is negligible.

Looking at the proposed GBSGA algorithm it can be seen that the complexity is low. It only needs:

- MAC operations: $2^{m}+13 m$,

- Test operations: $2 m$.

In Fig. 7 the differences between the number of cycles when both algorithms are used (the algorithm with and without calculating the permutations) are shown. At the decoder side, the differences are more significant because many permutations must be generated for the decoding process. It should be highlighted that this is the upper-bound for the complexity and it is rarely reached; In general, only a few GBS need to be generated at the decoding stage and thus the complexity is drastically reduced with respect to this upperbound. Besides, despite the complexity is slightly higher no look-up table is needed here and therefore there is a saving in memory as it can be seen in Table 3 . In this Table 3, the memory needed is assuming the GBS are stored in binary and therefore they need to be generated. If we want to avoid to compute GBS, the total memory must be increased by a factor of $2^{h}$.

A hybrid solution between choosing a particular number of sequences and generating them in real-time could also be used: storing a portion $(1 / 2,1 / 4, \ldots)$ of all wanted permutations and generating the others when they are needed. In this way, complexity is reduced $1 / 2,1 / 4$ and so forth, although in this case the memory requirements are increased twice, four times, etc. So there is a trade-off between memory and complexity. Additionally, another advantage is avoiding the storage of the CCK sequences since they can be real-time generated.

\section{Implementation}

The proposed Golay encoded OFDM-system has been implemented in a TMS320C6201@200MHz DSP from Texas Instruments to validate the design in a prototype. Two converters have been connected to the DSP in order to obtain the physical signal, the 12-bits dual Digital to Analog Converter (DAC) TLV5638 and the 12-bits Analog to Digital Converter (ADC) TLV 2541, both from Texas Instruments. The prototype has been scaled in some parameters as the number of sub-carriers, frequency bandwidth and carrier frequency from the typical values of an OFDM-based WLAN system [30], due to constrains in the development's platform. The number of sub-carriers was fixed to $N=32$, the bandwidth is $6.25 \mathrm{kHz}$ and the carrier frequency is $62.5 \mathrm{kHz}$. The proposed algorithm for real-time generation of the GBS was implemented at both sides, encoder and decoder, avoiding in this way the use of physical memory to store GBS. This memory was used to buffer the signal instead, alleviating therefore the complexity and time constrains for the other blocks at the receiver. The transmitter scheme is depicted in Fig. 8 where it can be observed that all the signal processing is carried out inside the DSP. In this figure, different blocks account for a variety of transmission operations: constellation mapping (Variable Mapper), OFDM modulation (IFFT), parallel to serial conversion $(\mathrm{P} / \mathrm{S})$ and insertion of the cyclic prefix $(\mathrm{CP})$.

On the other hand, in order to evaluate the PAR of the implemented system, a long transmission was used (about 4, 000 OFDM symbols) obtaining a PAR for the uncoded signal around $10 \mathrm{~dB}$ whereas PAR for the Golay encoded signal is close to $6 \mathrm{~dB}$. Although we theoretically expected a PAR limited to $3 \mathrm{~dB}$ after Golay encoding, it was proved in [31] that there is a difference between simulations and implementation of $3 \mathrm{~dB}$. This is because in simulations a low-pass equivalent signal representation is applied whereas for the implementation the real-valued signal is used. Then, our measurements in the prototype of a PAR around $6 \mathrm{~dB}$, for the Golay encoded signal, agree with the expected results. The noise error can be considered negligible. 

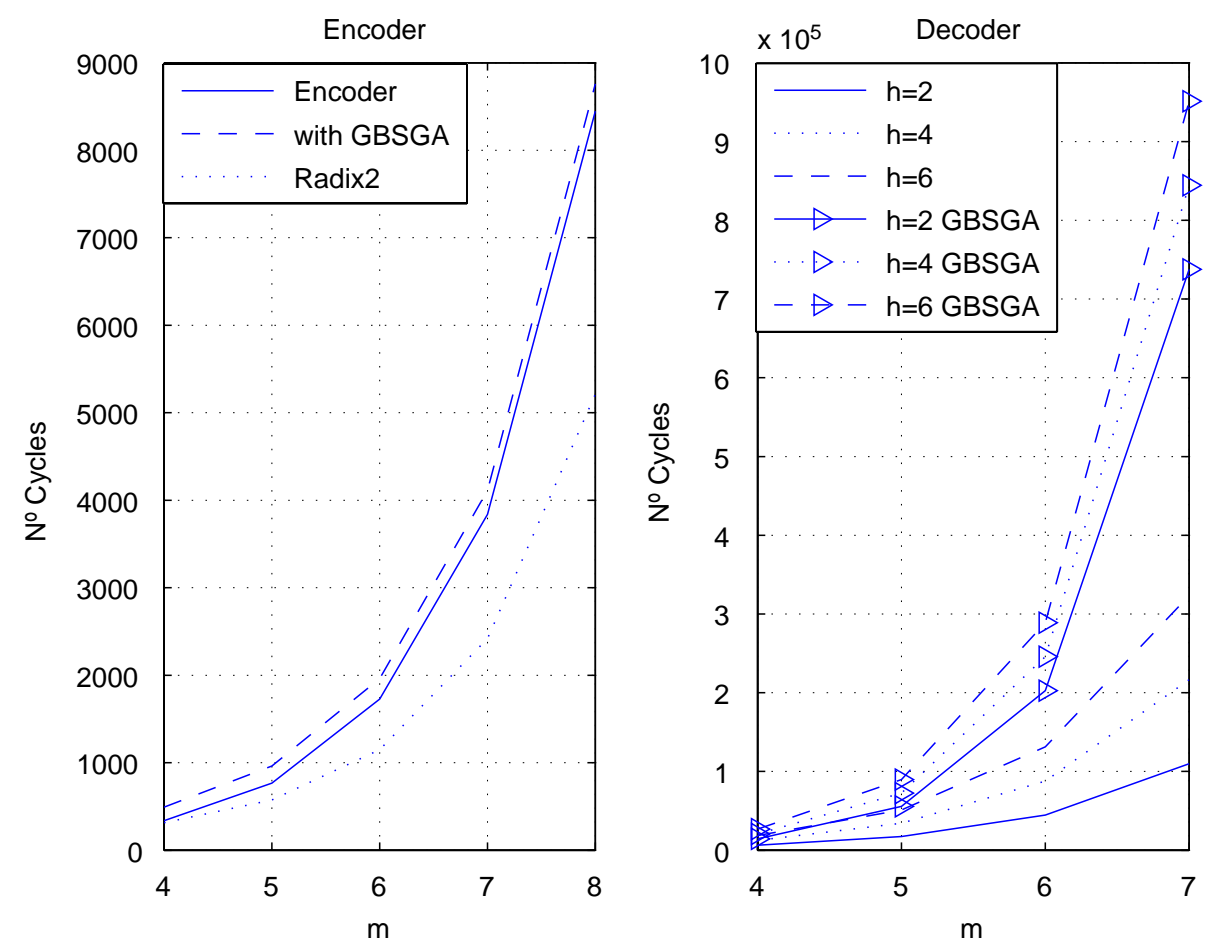

Fig. 7. Number of cycles with the real-time Golay Base Sequences Generation Algorithm (GBSGA).

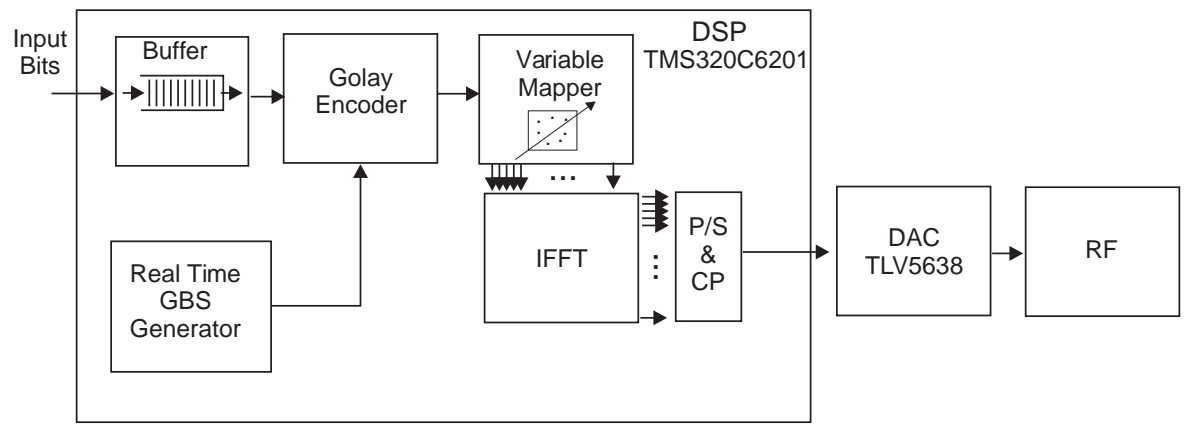

Fig. 8. Block diagram of the transmitter.

In Fig. 9 it can be seen that the uncoded OFDM output signal $^{4}$ exhibits large peaks whereas the Golay encoded OFDM signal shown in Fig. 10 presents less and lower peaks. Comparing both figures, a clear peak reduction can be observed in the signal after Golay encoding process. In Table 4 some numerical peaks percentages are shown in order to evaluate the peak reduction. The values are the percentage of the signal above the specific voltage for the uncoded and the encoded signal, respectively.

\footnotetext{
${ }^{4}$ The voltage range of the DAC is $[0 \sim 4.7] \mathrm{V}$. Therefore the DC component was fixed to $2.1 \mathrm{~V}$.
}

Table 4. Cumulative Distribution Function (CDF) for uncoded and encoded symbols

\begin{tabular}{lll}
\hline$>(V)$ & Uncoded $(\%)$ & Encoded $(\%)$ \\
\hline 2.2 & 34.68 & 31.12 \\
2.3 & 26.24 & 22.96 \\
2.4 & 19.28 & 15.68 \\
2.5 & 12.80 & 9.44 \\
2.6 & 8.80 & 4.88 \\
2.7 & 5.68 & 2.00 \\
2.8 & 3.16 & 0.36 \\
2.9 & 1.44 & 0 \\
3.1 & 0.68 & 0 \\
3.2 & 0.16 & 0 \\
\hline
\end{tabular}




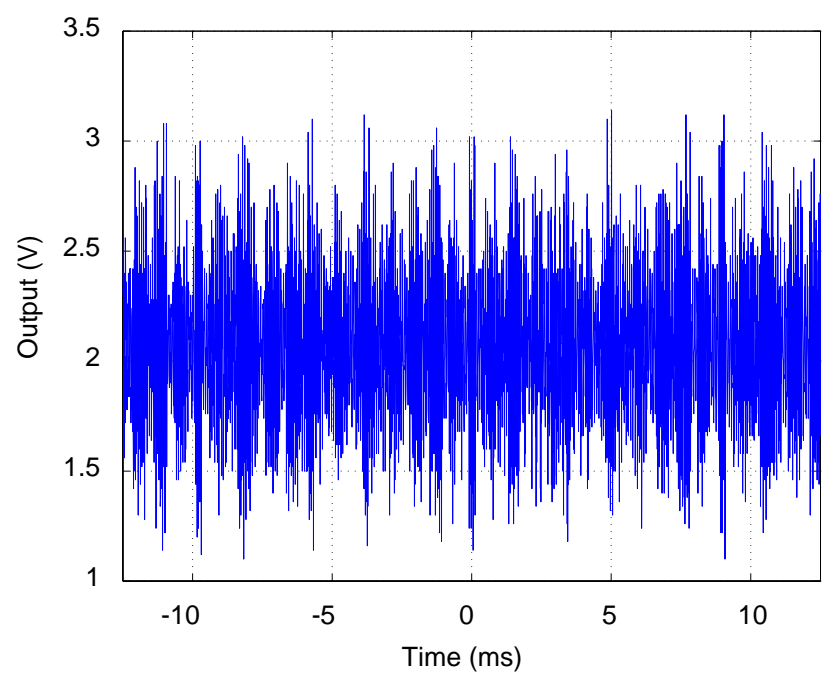

Fig. 9. OFDM output signal at the transmitter: uncoded.

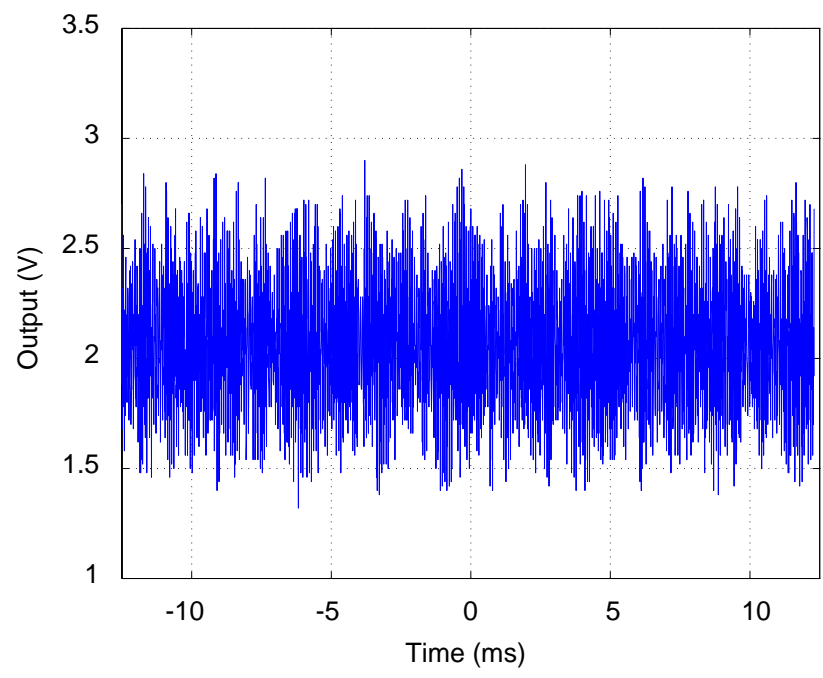

Fig. 10. OFDM output signal at the transmitter: Golay encoded.

\section{Conclusions}

OFDM is a good candidate to build next generation high bit rate WLANs but some implementation impairments such as its high peak-to-average power ratio must be solved to avoid the loss in efficiency in high power amplifiers. In this paper, the performance of CGS has been analyzed from the point of view of both PAR reduction and forward error correction. In this study it has been shown that for a low number of sub-carriers, as in WLAN environments, there is certain flexibility to select parameters $m$ and $h$ in order to fulfill system requirements. Moreover, a useful and efficient algorithm for real-time generation of these CGS has been proposed and analyzed. This way memory requirements for the implementation of CGS are reduced while the increase in complexity is negligible. Finally, an OFDM prototype with Golay encoding has been built and tested to validate their performance. In this prototype the real-time GBS Generation Algorithm was also implemented showing its feasibility.

The theoretical PAR for CGS was $3 \mathrm{~dB}$ whereas physical PAR obtained from the prototype was $6 \mathrm{~dB}$. The difference agrees with the fact that the real-valued signal was used instead of the low-pass equivalent. Besides their PAR reduction characteristics, these Golay sequences are able to correct channel errors providing FEC coding gain that improves the overall system performance.

\section{Acknowledgement}

The first author would like to thank Dr. J.A. Davis for his valuable help at the beginning of this work. This work has been partly funded by the Spanish government with projects MACAWI (TEC 2005-07477-c02-02) and MAMBO (UC3M-TEC-05-027). This paper was presented in part at the IEEE Mediterranean Electronic Conference MELECON'02, El cairo, Egypt.

\section{References}

[1] IEEE: WLAN Medium Access Control (MAC) and Physical Layer (PHY) specifications: high-speed physical layer in the $5 \mathrm{GHz}$ band. Technical report 802.11a. IEEE, June 2001.

[2] IEEE: WLAN Medium Access Control (MAC) and Physical Layer (PHY) specifications: high-speed physical layer in the $5 \mathrm{GHz}$ band. IEEE std $802.11 \mathrm{~g}-2003$ (amendment to IEEE std 802.11, 1999 edn. (reaff 2003) as amended by IEEE stds 802.11a-1999, 802.11b-1999, 802.11b-1999/cor 1-2001, and 802.11d-2001). Technical Report 802.11g. 2003.

[3] IEEE: IEEE 802.16a: Air interface for fixed broadband wireless access systems. amendment 2: MAC modifications and additional Physical Layer specifications for $2-11 \mathrm{GHz}$ (2003).

[4] Bingham JAC. Multicarrier modulation for data transmission: An idea whose time has come. IEEE Commun Mag 1990;28: 5-14.

[5] Armada AG, Calvo M. Phase noise and sub-carrier spacing effects on the performance of an OFDM communication system. IEEE Commun Lett 1998;2:11-3.

[6] Steendam H, Moeneclaey M. The effect of synchronisation errors on MC-CDMA performance. In: Proceedings of IEEE international Conference on communications. ICC, 1999. p. 1510-14.

[7] Moose PH. A technique for orthogonal frequency division multiplexing frequency offset correction. IEEE Trans Commun 1994;42:2908-14.

[8] Saarinen I, Lasanen M, Ruuska P, Girole G, Caverni A, Torregiani M, et al. Main approaches for the design of wireless indoor flexible high bit rate wind-flex modem architecture. In: Proceedings of IST mobile communications summit, Galway, Ireland, 2000. p. 51-6. 
[9] Awater G, van Nee DJR. Implementation of the Magic WAND wireless ATM modem. Proceedings IEEE international conference on communications, 1999. p. 1906-11.

[10] Merchán S, García A, García J. OFDM performance in amplifier non linearity. IEEE Trans Broadcasting 1998;4: 106-14.

[11] Suh C, Hwang C-S, Choi H. Preamble design for channel estimation in MIMO-OFDM systems. IEEE Proceedings Globecom, December 2003. p. 317-21.

[12] Davis JA, Jedwab J. Peak-to-Mean power control in OFDM, Golay complementary sequences and Reed-Muller codes. IEEE Trans Inf Theory 1999;45:2397-417.

[13] Sollenberger N. Peak-to-average power ratio reduction of an OFDM signal using partial transmit sequences. IEEE Commun Lett 2000;4:86-8.

[14] Jayalath A, Tellambura C. SLM and PTS peak-power reduction of OFDM signals without side information. IEEE Trans Wireless Commun 2005;4:2006-13.

[15] Tellado J, Cioffi J. Peak power reduction for multicarrier transmission. In: Proceedings IEEE global telecommunication conference (GLOBECOM). November, 1998, pp. 219-24.

[16] Cimini LJ Jr., Daneshrad B, Sollenberger N. Clustered OFDM with transmitter diversity and coding. Proceedings IEEE global telecommunication conference (GLOBECOM). November, 1996, p. 703-7.

[17] Edfords O, Borrallo JP. Peak power reduction for OFDM systems with orthogonal pilot sequences. IEEE Trans Wireless Commun 2006;5:47-51.

[18] Jeon W, Chang KH, Cho S. An adaptative data predistorter for compensation of nonlinear distortion in OFDM systems. IEEE Trans Commun 1997;45:1167-71.

[19] IEEE: Wireless LAN Medium Access Control (MAC) and Physical Layer (PHY) specifications: High-speed physical layer extension in the $2.4 \mathrm{GHz}$ band. IEEE $802.11 \mathrm{~b}$ (1999).

[20] Jeong W, Park H, Lee H, Hwang S. Performance improvement techniques for CCK-OFDM WLAN modem. IEEE Trans Consumer Electron 2003;49:602-5.

[21] Ochiai H, Imai H. On the Distribution of the peak-toaverage power ratio in OFDM Signals. IEEE Trans Commun 2001;49:282-9.

[22] Hunziker T, Bernhard UP. Evaluation of coding and modulation based on Golay complementary sequences for efficient OFDM transmission. In: Proceedings vehicular technology conference (VTC), May, 1998. p. 1631-35.

[23] Bell DA. Walsh functions and hadamard matrices. IEE Electron Lett 1966;2:340-1.

[24] Grant AJ, van Nee RD. Efficient maximum-likelihood decoding of Q-ary modulated Reed-Muller codes. IEEE Commun Lett 1998;2:134-6.

[25] Jiménez VPG, Fernández MS, Armada AG. Study and Implementation of Complementary Golay Codes for PAR reduction in OFDM Signals. In: Proceedings of IEEE Mediterranean electronical conference. MELECOM'02, May, 2002, p. 198-203.

[26] Chong CV, Venkataramani R, Tarokh V. A new construction of 16-QAM Golay complementary sequences. IEEE Trans Inf Theory 2003;49:2953-9.

[27] Chong CV, Venkataramani R, Tarokh V. Correction to a new construction of 16-QAM Golay complementary sequences. IEEE Trans Inf Theory 2004;50:1374.
[28] Instruments, T. TMS320C62x DSP Library Programmers's Reference. SPRU402b. Technical report, Texas Instruments, 2003.

[29] Knuth DE. Fundamentals algorithms. 3rd ed., Reading, Massachusetts: Addison-Wesley; 1997.

[30] Lobeira M, García A, Torres R, García JL. Parameter estimation and indoor channel modelling at $17 \mathrm{GHz}$ for OFDM-based broadband WLAN. In: Proceedings IST Mobile Summit (2000), 29-33.

[31] Tellado J. Peak-to-average power reduction. Dissertation. Standford University, September 1999.

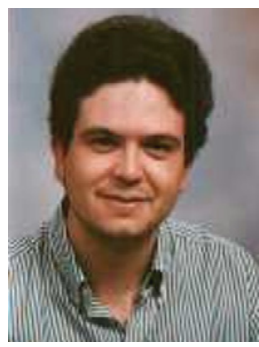

Víctor P. Gil Jiménez received the B.E. in Telecommunications with honors from University of Alcala in 1998 and the M.E. and Ph.D. degree both from the University Carlos III de Madrid in 2001 and 2005, respectively. $\mathrm{He}$ is with the Department of Signal Theory and Communications at the University Carlos III de Madrid as an Assistant Professor. He worked at the Spanish Antarctica Base in 1999 as

Communications Staff. He has participated in several national and international research projects related to Orthogonal Frequency Division Multiplexing (OFDM). He has performed research stays at University of Leeds, UK, and Chalmers Technical University, Sweden, in 2003 and 2004, respectively. In 1998 and 2006, he respectively received the best 'Master Thesis' and 'Ph.D. Thesis' awards from the Professional Association of Telecommunication Engineers of Spain. His research interests include multi-carrier communications and signal processing for wireless systems.

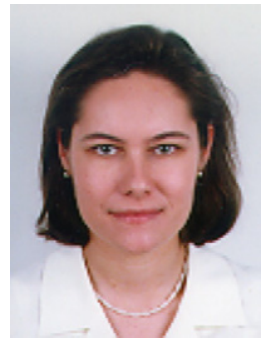

M. Julia Fernández-Getino García received the M.E. and Ph.D. degrees in telecommunication engineering, both from the Polytechnic University of Madrid, Spain, in 1996 and 2001, respectively. Currently, she is with the Department of Signal Theory and Communications of Carlos III University of Madrid, Spain, as an Associate Professor. From 1996 to 2001, she held a research position at the Department

of Signals, Systems and Radiocommunications of Polytechnic University of Madrid. She was on leave during 1998 at Bell Laboratories, Murray Hill, NJ, and visited Lund University, Sweden, during two periods in 1999 and 2000, and Politecnico di Torino, Italy, during 2003 and 2004. Her research interests include multicarrier communications, coding and signal processing for wireless systems. In 1998 and 2003, she respectively received the best 'Master Thesis' and 'Ph.D. Thesis' awards from the Professional Association of Telecommunication Engineers of Spain, and in 1999 and 2000, she was respectively awarded the 'Student Paper Award' and 'Certificate of Appreciation' at the IEEE International Conferences PIMRC'99 and VTC'00. In 2004, she was distinguished with the 'Ph.D. Excellence Award' from the Polytechnic University of Madrid. 


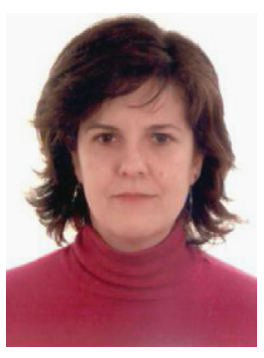

Matilde P. Sánchez Fernández got her Telecommunication Engineer degree from Technical University of Madrid in 1996, and her Doctor degree from Technical University of Madrid in 2001. She is an Assistant Professor at University Carlos III of Madrid, Spain since April 2000. Previously, she worked for Telefónica as a Telecommunication Engineer. She has performed several research stays at the Information and Telecommunication Technology Center in Kansas University and at BellLabs, New Jersey. Her current research interests are MIMO techniques, Turbo Codes, mobile communications, simulation and modelling of communication systems, and Adaptive Coding \& Modulation.

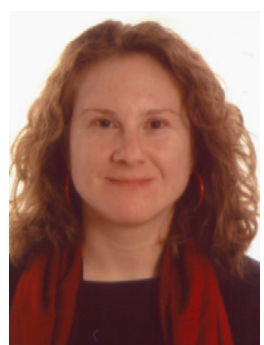

Ana García Armada received the Telecommunication Engineer degree and Ph.D. in Electrical Engineering both from the Polytechnic University of Madrid (Spain) in July 1994 and February 1998, respectively. She is currently working as an Associate Professor at the University Carlos III de Madrid, Spain where she has occupied several management positions. She has participated in several national and international research projects, most of them related to Orthogonal Frequency Division Multiplexing (OFDM). She is co-author of four books on wireless communications and signal processing. She has published 13 papers in international journals and more than 40 papers in international conferences. She has contributed to international organizations such as ITU and ETSI. She has performed research stays in ESA-ESTEC, Kansas University, Stanford University and Bell Labs. Her research interests are simulation of communication systems, multicarrier and MIMO techniques. 